\title{
Analyse des récentes sécheresses du Sud-Ouest
}

\author{
G. Couzy \\ Ingénieur en Chef des Ponts et Chaussées \\ Chef du Service Navigation de Toulouse \\ Délégué du Bassin Adour-Garonne
}

\author{
A. Le Vouedec, \\ B. Jarry \\ Délégation de bassin Adour-Garonne
}

Le bassin Adour-Garonne qui s'étend sur 6 Régions (2 intégralement et 4 en partie) intéresse 25 départements.

Le réseau hydrographique est composé d'un grand axe (la Garonne) et de rivières affluentes ou non à celui-ci (le Tarn, le Lot, l'Adour, l'Ariège, la Dordogne, la Charente, l'Aveyron).

Ces rivières sont caractérisées par des débits très variables puisque la Garonne, à la station de Lamagistère en amont d'Agen, atteint des débits moyens de référence variant de 700 à $130 \mathrm{~m}^{3} / \mathrm{s}$.

Les prélèvements en eau dans le bassin sont estimés en 1988 à 2,4 milliards de $\mathrm{m}^{3}$.

Pour satisfaire les multiples besoins en eau (navigation, eau potable, irrigation, réalimentation des rivières), un ensemble de réservoirs totalisant environ 320 millions de $\mathrm{m}^{3}$ a été réalisé depuis la fin du $\mathrm{XVII}^{\mathrm{e}}$ siècle. Ils se répartissent comme suit :

— grands ouvrages (supérieurs à 2 millions) : 190 millions de $\mathrm{m}^{3}$;

— retenues collinaires : 130 millions de $\mathrm{m}^{3}$.

A cela il convient d'ajouter les réserves hydroélectriques d'EDF et de la SNCF qui représentent 2500 millions de $\mathrm{m}^{3}$ et qui sont réservées à la production électrique.

L'économie du bassin est dominée par l'agriculture.

Afin de régulariser la production agricole et d'être plus compétitif dans le contexte européen, les surfaces irriguées ont quasiment triplé entre 1970 et 1985 , passant de 132000 hectares en 1970 à 360000 hectares en 1985.

\section{Analysis of the recent droughts in the south-west of France}

The drought of Autumn 1986 was all the more difficult since it followed that of 85 which lasted until quite late. Winter and Spring of 86 saw a return to normal rainfall but the month of May saw the beginning of a new dry period which was to last until the beginning of Winter.

The succession of these two exceptionnally dry periods led to rapid consummation of water level reserves from 1986 onwards. In 1986 the phenomenon is more rigourous than in 1985 and much more so than in 1976: You have to go back to 1949 to find an equivalent drought.

This led the Basin Delegate to set up a "crisis cell » to follow the development of the phenomenon and to propose to the Basin Coordinating Prefect a series of actions followed by effects : reduction of the flow delivered by the Neste canal, limitation of the supply flow rate of the canal parallel to the Garonne, releasing of water from EDF reserves, and finally stopping of pumping for irrigation.

The succession of these two worrying periods also enabled all partners to become aware of the problems, and triggered off the development programme approved of by the Committee for the Ardour-Garonne Basin, and plans for construction work over 10 years to provide a total capacity of 300 million cubic meters for a total cost of 1480 million francs. 
Ainsi en 1985 la répartition des usages de l'eau dans le bassin Adour-Garonne était la suivante :

\begin{tabular}{|c|c|c|c|c|c|}
\hline Usages & : Eau potable & $\vdots$ & Eau industrielle & $\vdots$ & Irrigation \\
\hline $\begin{array}{l}\text { Besoin en eau en } \\
\text { en millions de } \mathrm{m}^{3}\end{array}$ & 620 & : & 1200 & $\vdots$ & 538 \\
\hline $\begin{array}{l}\text { Volume d'eau } \\
\text { consommée (non } \\
\text { restituée au cours } \\
\text { deau en millions } \\
\text { de } \mathrm{m}^{3} \text { ) }\end{array}$ & $\begin{array}{c}\vdots \\
\vdots \\
\vdots\end{array}$ & $\begin{array}{l}\vdots \\
\vdots \\
\vdots \\
\vdots\end{array}$ & 87 & $\vdots$ & 377 \\
\hline $\begin{array}{l}8 \text { de leau consorn- } \\
\text { mée par rapport aux } \\
\text { besoins }\end{array}$ & $\vdots$ & $\vdots$ & 78 & & $70 \%$ \\
\hline
\end{tabular}

Pour mieux cerner le contexte dans lequel se produisent les sécheresses successives que connaît le bassin Adour-
Garonne depuis un lustre, il faut signaler que, outre la satisfaction des usages domestiques, industriels et d'irrigation, l'eau est également un élément primordial pour le maintien d'autres activités telles que : tourisme, navigation et une action d'envergure menée par les pêcheurs: le programme de restauration des poissons migrateurs.

Ainsi, en période de pénurie, les administrations et usagers de l'eau doivent-ils opérer des choix draconiens entre ces différents usages, ayant des répercussions économiques et psychologiques pour l'usager comme pour les collectivités.

Nous allons examiner les diverses sécheresses subies par le bassin Adour-Garonne en 1985, 1986 et 1989.

Nous nous attarderons à 1989 où, compte tenu de l'expérience des années antérieures et de la nature de la sécheresse, la conduite des opérations a été menée de façon légèrement différente.
Alors que les précipitations sur l'ensemble du bassin ont été normales jusqu'en juin, elles sont de plus en plus déficitaires à partir de juillet et exceptionnellement faibles, voire inexistantes en de nombreux endroits en septembre.

Les effets de ce déficit pluviométrique se font particulièrement sentir à la fin de l'été jusqu'à la première quinzaine de novembre.

La pluviométrie de l'année 1985 est la suivante à Toulouse-Blagnac (en $\mathrm{mm}$ ) :

\begin{tabular}{l|cccccccccccc|c} 
Mols & $J$ & F & M & A & $M$ & J & J & A & S & O & N & D & TOTAL \\
\hline $\begin{array}{l}\text { Moyenne } \\
\text { 1931-1960 }\end{array}$ & 49 & 53 & 53 & 50 & 75 & 61 & 44 & 54 & 64 & 45 & 51 & 67 & 666 \\
\hline 1985 & 43 & 72 & 80 & 67 & 81 & 75 & 58 & 13 & 1 & 31 & 42 & 53 & 616 \\
\hline $\begin{array}{l}\text { Rapport i ia } \\
\text { moyenne 8 }\end{array}$ & 88 & 136 & 151 & 134 & 108 & 123 & 132 & 24 & 1,5 & 69 & 82 & 79 & 92 \\
\hline
\end{tabular}

L'analyse de la pluviométrie relevée à Auch pour la période 1925-1985 donne pour les mois de juin à octobre les chiffres suivants :

\begin{tabular}{|c|c|c|c|c|}
\hline & \multicolumn{4}{|c|}{ TOTAL DES RELEVES PLUVIOKETRIOUES (en mm) } \\
\hline & $\begin{array}{r}\text { Sur } 5 \text { mols } \\
\text { Juin i Octobre }\end{array}$ & $\begin{array}{c}\text { Sur } 4 \text { mois } \\
\text { : vuin à Septambre }\end{array}$ & $\begin{array}{c}\text { Sur } 4 \text { mois } \\
\text { veiliot i Oetoors }\end{array}$ & $\begin{array}{c}\text { Sur } 3 \text { mois } \\
\text { Jullet i Septemore }\end{array}$ \\
\hline $\begin{array}{l}\text { Total depassib } \\
1 \text { ennie sur } 2\end{array}$ & 271 & 200 & 198 & 143 \\
\hline $\begin{array}{l}\text { Total depessse } \\
9 \text { annees sur } 10\end{array}$ & 172 & 110 & 133 & 81 \\
\hline minimum observe & 94 (en 1985) & 75 (en 1985) & 44 (en 1985) & 25 (en 1985) \\
\hline
\end{tabular}

Ainsi, du fait de la chute brutale des précipitations en août, 1985 s'avère l'année la plus sèche pour les quatre mois d'été depuis 60 ans.
On observe également une chute brutale des débits des cours d'eau puisqu'à la station de Lamagistère sur la Garonne nous avons :

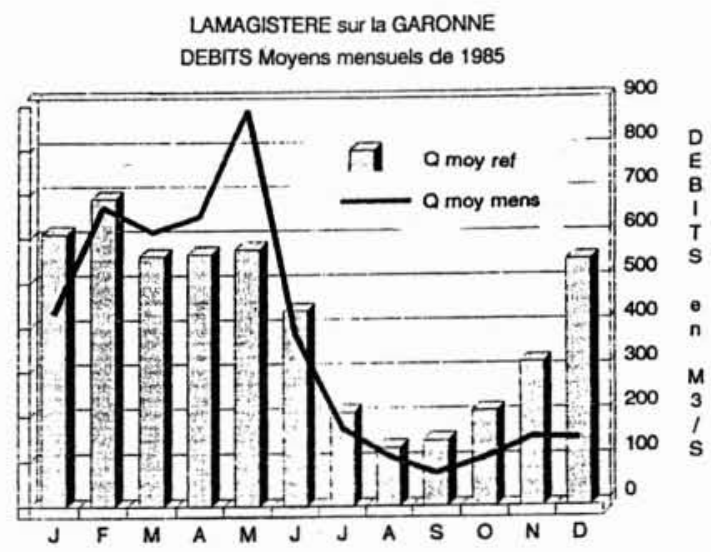

Le débit moyen journalier minimal était atteint le 15 septembre 1985 avec $46 \mathrm{~m}^{3} / \mathrm{s}$ pour une moyenne de référence de $152 \mathrm{~m}^{3} / \mathrm{s}$

La sécheresse s'avère être de fréquence plus que quinquennale sur la Garonne.

A cela il convient d'ajouter des températures élevées aggravant le phénomène d'évapotranspiration et l'effet de l'absence de pluies.

Les conséquences en ont été :

- records de consommation pour l'irrigation dans les vallées du Lannemezan: $1750 \mathrm{~m}^{3} /$ hectare en moyenne avec des niveaux supérieurs à $2000 \mathrm{~m}^{3} /$ hectare dans certains secteurs ; 
- ralentissement des activités de certaines industries (notamment délainage) ;

- réquisition des eaux de la Neste.

Cependant, les conséquences ont été atténuées car cette sécheresse de 1985 a été tardive (fin de l'été et automne) et a été suivie d'un printemps bien arrosé qui a permis une bonne alimentation des nappes et des cours d'eau et une bonne reconstitution des réserves de soutien d'étiage.

Face à la situation pluviométrique et hydrologique le 27 septembre 1985, le Préfet de la Région Midi-Pyrénées créait une commission chargée du suivi de la gestion des ressources en eau des bassins supérieurs de la Neste (Lannemezan) et de la Garonne avec l'appoint des réserves d'EDF.
Cette commission se réunissant chaque semaine a traité la prise des décisions suivantes:

- diminution progressive des lâchures du système Neste : - diminution du débit d'alimentation du canal latéral à la Garonne ;

- le 26 octobre, déstockage à partir des réserves $E D F$, pour assurer un débit supplémentaire de $2 \mathrm{~m}^{3} / \mathrm{s}$ sur la Neste ;

- le 30 octobre: réquisition des eaux de la Neste par arrêté préfectoral.

Le 6 novembre, à la suite de précipitations généralisées, la situation redevient normale.
Nous venons de voir que l'année 1985 avait été caractérisée par un déficit pluviométrique en été et en automne.

L'hiver suivant et le début du printemps 1986 ont été marqués par une pluviométrie "normale». Mais au mois de mai débute une nouvelle période de déficit pluviométrique qui va s'accentuer et se poursuivre jusqu'au mois de septembre.

Un bilan établi à la fin du mois de juillet fait ressortir un déficit pluviométrique général sur le Sud-Ouest, les zones les plus défavorisées se situent en Midi-Pyrénées, au nord des Landes et au sud de la Charente-Maritime.

Les quelques précipitations orageuses et localisées du mois d'août 1986 n'ont pas modifié la situation.

Le tableau ci-après donne la pluviométrie $(\mathrm{en} \mathrm{mm}$ ) à Toulouse-Blagnac de août 1985 à juillet 1986 et la comparaison à la moyenne normale sur 30 années (1931-1960) :

\begin{tabular}{|c|c|c|c|c|c|c|c|c|c|c|c|c|c|}
\hline is & $A$ & s & 0 & $\mathrm{~N}$ & D & J & f & m & A & $m$ & J & J & TOTA \\
\hline tioned & 54 & 64 & 45 & 51 & 67 & 49 & 53 & 53 & so & 75 & 61 & 44 & 666 \\
\hline $1985 / 1986$ & 13 & 1 & 31 & 42 & 53 & 87 & 49 & 45 & 64 & 43 & 18 & 3 & 44 \\
\hline voort: & 24 & 1.5 & 69 & 82 & 79 & 177 & 92 & 85 & 128 & 57 & $x$ & 6 & 67 \\
\hline
\end{tabular}

L'analyse sur les trois mois de mai-juin et juillet 1986 donne une pluviométrie de $64,1 \mathrm{~mm}$, soit $35 \%$ de la normale, et une comparaison aux années les plus sèches pour ces trois mois pendant la période 1947-1986 donne le classement suivant :

\begin{tabular}{|c|c|c|}
\hline MAI + JUIN + JUILLET & $:$ & Pluies $(\mathrm{mm})$ \\
\hline 1986 & $:$ & 64,1 \\
\hline 1950 & : & 74,9 \\
\hline 1976 & & 80.7 \\
\hline
\end{tabular}

Si au regard de la pluviométrie d'août 1985 à juillet 1986 le déficit varie de 10 à $30 \%$ selon les stations, ce qui correspond à une période de retour comprise entre 5 et 10 ans, en revanche la pluviométrie printanière présente un déficit de 50 à $70 \%$; la période de retour est comprise entre 50 et 60 ans.

Du fait de la succession des deux sécheresses 1985 et 1986, le niveau des nappes du printemps 1986 n'a pas retrouvé son niveau normal.

Le graphique suivant donne la variation du niveau piézographique de la nappe alluviale de la Garonne à St Porquier (en aval de Toulouse) :

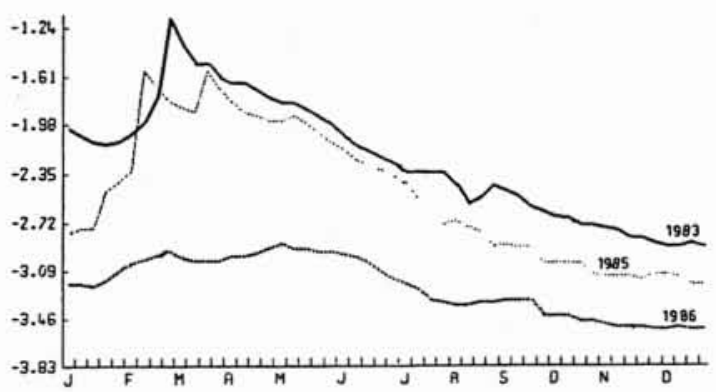

Bien que la pluviométrie du début de l'année 1986 ait été sensiblement normale, le déficit de la nappe de la Garonne qui s'est amorcé à l'issue de la sécheresse de l'automne 1985 s'est prolongé et accentué en 1986 pour atteindre un abaissement de $45 \mathrm{~cm}$ entre septembre 1985 et septembre 1986.

La comparaison des débits des sources du Quercy et des sources karstiques pyrénéennes pour les années particulièrement sèches que sont 1976-1985-1986 donne :

\begin{tabular}{|c|c|c|c|}
\hline & $\begin{array}{l}\text { Sourca de CATOS } \\
\text { QueRCY }\end{array}$ & $\begin{array}{l}\text { Source Ge BAGET } \\
\text { PYRENEES }\end{array}$ & $\begin{array}{c}\text { Source de FONIESTOFBES } \\
\text { PYRENESS }\end{array}$ \\
\hline $\begin{array}{l}\text { Moyenne de } \\
\text { SEPTERBRE sur } \\
20 \text { ans }\end{array}$ & 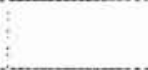 & $200 \mathrm{l} / \mathrm{s}$ & $1100 \mathrm{l} / \mathrm{s}$ \\
\hline SEPTEMERE 1976 & $\vdots$ & $124 \mathrm{l} / \mathrm{s}$ & $930 \mathrm{k} / \mathrm{s}$ \\
\hline SEPTEMBRE I9BS & $25 \mathrm{l} / \mathrm{s}$ & $83 \mathrm{l} / \mathrm{s}$ & $889 \mathrm{l} / \mathrm{s}$ \\
\hline SEPTEMERE 1986 & $1 / s$ & $73 \mathrm{l} / \mathrm{s}$ & $872 \mathrm{l} / \mathrm{s}$ \\
\hline
\end{tabular}


Ainsi, le printemps 1986 débutait-il pour les nappes, mais aussi pour les sources, avec un déficit. Ce déficit des réserves souterraines se répercute sur les cours d'eau en période d'étiage.

En juin, avant le début des campagnes d'irrigation, la situation est la suivante pour les cours d'eau, les nappes et les réserves de soutien d'étiage:

\section{ETAT DES RESERYES AU 15 JUIN 1986}

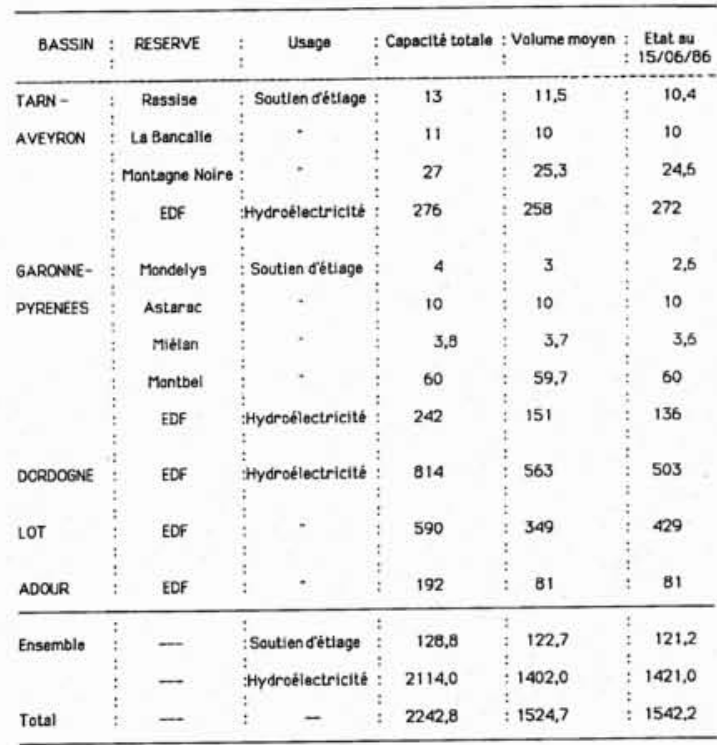

Débits de la GARONNE a PORTET (amont de TOULOUSE et LAMAGISTERE (amont d"AGEN)

\begin{tabular}{|c|c|c|c|c|c|c|c|}
\hline STATION & Debits $\mathrm{en}^{3} \mathrm{~m}^{3} / \mathrm{s}$ & $\mathrm{J}$ & $F$ & $M$ & A & $M$ & $J$ \\
\hline \multirow[t]{2}{*}{ LAMAGISTERE } & $\begin{array}{l}\text { Debit moyen } \\
\text { de refference }\end{array}$ & 610 & 690 & 560 & 570 & 575 & 434 \\
\hline & $\begin{array}{l}\text { Débit moyen } \\
\text { : mensuel an } 1986\end{array}$ & 479 & 620 & 535 & 765 & 605 & 254 \\
\hline \multirow{2}{*}{ PORTET } & $\begin{array}{l}\text { Debitt moyen } \\
\text { de rérérence }\end{array}$ & 196 & 219 & 210 & 269 & 739 & 301 \\
\hline & $\begin{array}{l}\text { Debit moyen } \\
\text { : mensual an } 1986\end{array}$ & 210 & 264 & 224 & 217 & 320 & 163 \\
\hline
\end{tabular}

Niveau de la nappe à ST PORQUIER (nappe alluviale de la GARONNE)

\begin{tabular}{|c|c|c|c|c|c|c|}
\hline & $\vdots$ & $F$ & $M$ & A & $M$ & J \\
\hline HAUTEUREN 1983. & $-2,06$ & $-2,04$ & $-1,36$ & $-1,64$ & $-1,80$ & -2.12 \\
\hline HAUTEUREN $1986 \ldots . . . . . . . . . . . . .$. & $\vdots \begin{array}{ll}\vdots \\
\vdots\end{array}-18$ & $-3,02$ & $-2,98$ & $-2,96$ & $-2,90$ & -2.96 \\
\hline
\end{tabular}

En juin, malgré les faibles précipitations des deux mois précédents, la situation ne laisse pas présager une sécheresse exceptionnelle.

Mais la conjonction de la sécheresse qui est intervenue dès le début du cycle végétatif des principales cultures d'été, de l'importance de l'évapotranspiration des plantes et de la faiblesse des précipitations estivales a provoqué un épuisement rapide des réserves en eau des sols et l'apparition de déficits hydriques sévères pouvant entraîner de lourdes pertes de production pour les agriculteurs non équipés de système d'irrigation.

C'est pourquoi l'irrigation a commencé dès juillet de manière importante. Le record de consommation observé dans les vallées du Lannemezan (système Neste) est battu en 1986 puisque l'on atteint en moyenne $2100 \mathrm{~m}^{3} /$ hectare (1750 $\mathrm{m}^{3} /$ hectare en 1985).

Les réserves agricoles et de soutien d'étiage ont donc été sollicitées de manière précoce et intensive comme le montre le graphique ci-après (sous-bassin : Neste) :

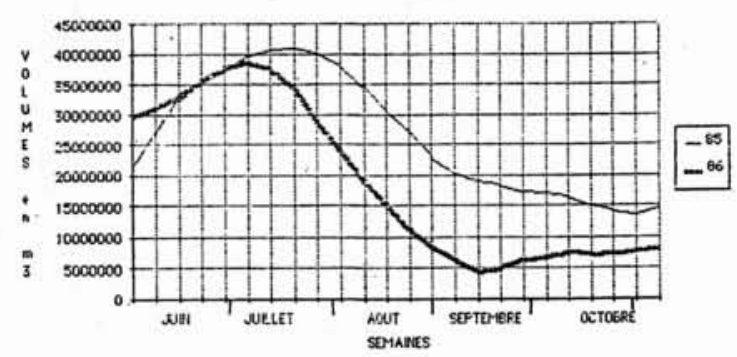

La pluviométrie de l'été 1986 est la suivante à ToulouseBlagnac :

\begin{tabular}{|c|c|c|c|c|c|c|c|}
\hline MOIS & $\vdots$ & JUIN & NILLET & AOUT & SEPTERBRE & OCTOBRE & NOVETBPE \\
\hline $\begin{array}{l}\text { Moyenne } \\
\text { (1953-1982) }\end{array}$ & $:$ & 64,1 & 46,4 & 47.2 & 57 & 52.2 & 53.1 \\
\hline 1986 & $:$ & 17.8 & 3,4 & 30,7 & 42.2 & 21.8 & 37.1 \\
\hline $\begin{array}{l}\text { Rasport b ia } \\
\text { moyenno \& }\end{array}$ & 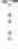 & 28 & 7 & 82 & 74 & 42 & 70 \\
\hline
\end{tabular}

A Tarbes, nous avons le tableau suivant:

\begin{tabular}{|c|c|c|c|c|c|c|c|}
\hline MOS & $:$ & JUIN & SUILLET & AOUT & SEPTETERE & OCTOGPE & NOVEMBRE \\
\hline $\begin{array}{l}\text { Moyenne } \\
\text { (1953-1982) }\end{array}$ & $\vdots$ & 8,8 & 53 & 66 & 81 & Bo & 91 \\
\hline 1986 & $\vdots$ & 24,0 & 7,8 & 28.3 & as, 8 & 67 & 95,6 \\
\hline $\begin{array}{l}\text { Rasopert i is } \\
\text { mayenne } 8\end{array}$ & $\vdots$ & 28 & 15 & 43 & 106 & 83 & 105 \\
\hline
\end{tabular}

Les réserves diminuent rapidement, les nappes souterraines accusent le choc de la sécheresse de 1985, les cours d'eau se retrouvent à l'été 1986 fortement sollicités par les prélèvements, avec peu d'alimentation. 
Les débits vont donc aller décroissants et les débits suivants sont enregistrés durant l'été 1986 à Lamagistère et Portet sur la Garonne :

\begin{tabular}{|c|c|c|c|c|c|c|c|c|}
\hline Stations & Mols & UIN & JULLET & AOUT & SEPTEM. & œCTOBPE & Noverepe: & $\begin{array}{l}\text { Debit moyen } \\
\text { Journalier } \\
\text { minimum }\end{array}$ \\
\hline \multirow{2}{*}{$\begin{array}{l}\text { LAMA- } \\
\text { GISTERE }\end{array}$} & $\begin{array}{l}\text { Debit } \\
\text { moyen de } \\
\text { retererence } \\
\text { en m3/s }\end{array}$ & 434 & 206 & 129 & 145 & 209 & 319 & \multirow{2}{*}{$\begin{array}{l}35,6 \mathrm{m3} / \mathrm{s} \\
25 \text { AOUT }\end{array}$} \\
\hline & $\begin{array}{l}\text { Dtbit } \\
\text { moyen } \\
\text { mensuel } \\
\text { an m3/s }\end{array}$ & 254 & 109 & 54.5 & 94.5 & 134 & 252 & \\
\hline \multirow[t]{2}{*}{ PCRTET } & 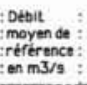 & 301 & 150 & 96,5 & 94,5 & 100 & 131 & \multirow[t]{2}{*}{$\begin{array}{l}26,6 \mathrm{~m} 3 / \mathrm{s} \\
10\end{array}$} \\
\hline & 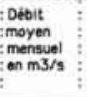 & 163 & 72 & 40,9 & 58,5 & 62.5 & 85 & \\
\hline
\end{tabular}

Par ailleurs, un débit instantané de $13 \mathrm{~m}^{3} / \mathrm{s}$ a été observé à l'aval de Toulouse le 19 août, après le prélèvement de $6 \mathrm{~m}^{3} / \mathrm{s}$ du Canal latéral à la Garonne.

Face à cette situation, l'Administration se mobilise fin juillet :

- le 7 août, une procédure "Calamités Agricoles " est ouverte pour les départements de l'Aveyron, de l'Ariège, de la Haute-Garonne, du Gers, du Lot, du Lotet-Garonne, du Tarn et du Tarn-et-Garonne.

- le 8 août, à l'initiative du Délégué de Bassin, les Services de différentes Administrations se réunissent pour examiner les mesures à prendre et informer le Préfet de la Région Midi-Pyrénées.

Cette cellule de crise se réunit toutes les semaines et examine l'évolution de la situation.

- Le 20 août: On constate une dégradation générale de la situation. On a mesuré un débit instantané de $13 \mathrm{~m}^{3} / \mathrm{s}$ à l'aval de Toulouse.

Le débit moyen journalier à la station de Loubéjac sur l'Aveyron est $1,9 \mathrm{~m}^{3} / \mathrm{s}$ alors que le débit moyen de référence est de $11,6 \mathrm{~m}^{3} / \mathrm{s}$.

Il est proposé de réduire le débit du canal de la Neste de $11 \mathrm{~m}^{3} / \mathrm{s}$ à $7 \mathrm{~m}^{3} / \mathrm{s}$. On envisage d'interdire les irrigations en Tarn-et-Garonne.

- le 22 août: Le Préfet de la Région de Midi-Pyrénées prend des mesures suivantes: demande de lâchures de $6 \mathrm{~m}^{3} / \mathrm{s}$ depuis le barrage de Montbel ; demande aux Préfets de Tarn-et-Garonne, Tarn, Aveyron, Gers, Lotet-Garonne d'interdire les irrigations à partir de la Garonne, du Tarn, de l'Aveyron et des rivières réalimentées par le système Neste ;

- le 27 août : Le département de la Haute-Garonne est déclaré sinistré ;

- le 2 septembre : le Préfet du Gers interdit les pompages à compter du 18 septembre ;

- le 5 septembre: Réunion de la " cellule de crise " : EDF soutient les débits de la Garonne par des lâchures de ses réserves ; on envisage de réduire le débit réservé de la Neste à $2 \mathrm{~m}^{3} / \mathrm{s}$;
- le 12 septembre: Le Préfet du Tarn-et-Garonne interdit les pompages à compter du 18 septembre ;

- le 15 septembre: Arrêté préfectoral d'interdiction de pompage dans le département de la Haute-Garonne à compter du 18 septembre;

- le 18 septembre: Arrêté préfectoral d'interdiction de pompage dans le département des Hautes-Pyrénées à compter du 18 septembre;

- le 25 septembre : Devant la situation critique des rivières réalimentées par le système Neste, les Préfets des Hautes-Pyrénées et de la Haute-Garonne demandent des lâchures supplémentaires à partir des réserves d'EDF; - le 23 octobre: Réquisition des eaux de la Neste. Le débit réservé est fixé à $1,5 \mathrm{~m}^{3} / \mathrm{s}$.

Le déficit en eau a duré tout l'automne et s'est maintenu jusqu'au début de l'hiver. La situation n'était plus alors aussi préoccupante du fait des arrêts d'irrigation et des températures moindres.

Le bilan de la sécheresse 1986 peut se traduire par quelques chiffres :

- en Ariège, les pertes de rendement sont de $15 \%$ sur le blé, $50 \%$ sur le maïs et le tournesol ;

- dans l'Aveyron, les pertes de rendement sur les herbages varient de 40 à $80 \%$, le blé et le maïs n'étant pas récoltés dans certaines régions ;

- en Haute-Garonne les pertes sur les prairies varient de $20 \%$ pour la première coupe à $100 \%$ pour la troisième coupe. Les dégâts subis par les cultures d'été irriguées $(30 \%)$ sont deux fois moins importants que les pertes des cultures non irriguées $(60 \%)$. Au total l'agriculture de la Haute-Garonne perd 189 millions de francs en production végétale ;

— dans le Gers les pertes sont estimées à 7,5 millions de francs.

Afin d'aider les 50000 agriculteurs sinistrés sur les 80000 que compte la région Midi-Pyrénées, le Conseil Régional débloque une enveloppe de 5 millions de francs qui vient s'ajouter aux aides et subventions débloquées par l'Etat dans le cadre du plan Sécheresse.

L'ensemble des mesures prises pour les agriculteurs (éleveurs et céréaliers) victimes de la sécheresse représente sur le plan national une enveloppe 1,3 milliard de francs qui met à contribution l'Etat ( 775 millions de francs), la Banque Verte (445 millions de francs) et le Fonds de Solidarité céréaliers-éleveurs unigraines ( 80 millions de francs). Elles portent sur:

- la fourniture de 400000 tonnes d'orge, dont 200000 sont distribuées gratuitement aux éleveurs les plus touchés ;

- l'aide au transport pour les céréales fourragères autres que ces 400000 tonnes;

- les aides financières: prêts calamités pour perte de récoltes, prises en charge des annuités des prêts bonifiés, dispositions spéciales pour les agriculteurs en situation sociale particulière. 
Les sécheresses de 1985 et de 1986 n'ont pas été suivies, en 1987 et 1988, d'événement très marquant. On peut seulement relever qu'en 1988 il y eut d'abondantes pluies printanières qui ont permis d'aborder l'été 1988 sans crainte.

A partir d'août 1988 les précipitations se font rares et l'examen de la pluviométrie à Toulouse-Blagnac explique les inquiétudes qui ont germé dans le Sud-Ouest au début de l'année 1989.

\begin{tabular}{|c|c|c|c|c|c|c|c|c|c|c|c|c|}
\hline MOIS & $J$ & M & A & M & $J$ & $\mathrm{~J}$ & A & s & 0 & $\mathrm{~N}$ & D & TOTAL \\
\hline Moyenne & 57 & $52 \quad 57$ & 56 & 71 & 64 & 47 & 47 & 56 & 50 & s5 : & 59 & 671 \\
\hline 1988 & 102 & $54 \quad 100$ & 171 & 121 & 105 & 63 & 17 & 30 & 23 & 19 & 23 & 828 \\
\hline 1989 & 20 & $94 \quad 63$ & 120 & & & & & & & & & \\
\hline $\begin{array}{l}\text { Rapport a la } \\
\text { moyenne } 8 \\
\text { Wuillet } 1988\end{array}$ & \begin{tabular}{|c|}
35 \\
- Mars \\
\end{tabular} & $\begin{array}{l}180 \quad 110 \\
s \text { 1989) }\end{array}$ & 214 & & & 134 & 36 & 53 & 46 & 34 & 39 & \\
\hline
\end{tabular}

Ce déficit pluviométrique de l'automne et l'hiver 1988 fut tout d'abord signalé par les Services de la Météorologie nationale et recevait de la part des médias nationales un vaste écho qui pouvait émouvoir la population.

Le bilan établi en février 1989 montre que dans l'ensemble du bassin Adour-Garonne le volume de pluie tombée depuis le $1^{\text {er }}$ septembre 1988 est inférieur de $70 \%$ à la moyenne.

Après avoir scruté le ciel, les observateurs ont baissé la tête et ont regardé sous les ponts. La situation n'était guère plus brillante et autant il manquait de l'eau en haut, autant il semblait en manquer en bas.

L'analyse du débit des cours d'eau illustre bien ce déficit. Nous donnons, à titre d'exemple, les valeurs des débits moyens mensuels observés aux stations de Lamagistère et de Portet.

\begin{tabular}{|c|c|c|c|c|}
\hline & MOIS & $\vdots$ & JANVIER 1989 & FEVRIER 1989 \\
\hline \multirow{2}{*}{ LAMAGISTERE } & $\begin{array}{l}\text { Débit moyen de } \\
\text { :réference } \mathrm{m} 3 / \mathrm{s}\end{array}$ & $\vdots$ & 610 & 690 \\
\hline & $\begin{array}{l}\text { Deolt moyen } \\
\text { :mensuel m3/s }\end{array}$ & $\vdots$ & 92 & 124 \\
\hline \multirow{2}{*}{ PORTET } & $\begin{array}{l}\text { Débit moyen de } \\
\text { :réference } \mathrm{m} 3 / \mathrm{s}\end{array}$ & $\vdots$ & 196 & 219 \\
\hline & $\begin{array}{l}\text { Débit moyen } \\
\text { mensuel m3/s }\end{array}$ & $\begin{array}{c}\vdots \\
\vdots\end{array}$ & 64,5 & 60 \\
\hline
\end{tabular}

Ainsi le déficit pluviométrique se traduisait en février 1989 de la façon suivante :

- les stocks de neige, qui constituent une part importante des débits de printemps, sont les plus bas connus en trente ans, le déficit est de $70 \%$ par rapport à la moyenne ; - les réserves en eau des sols, normalement saturés en février, ont un déficit de $70 \%$ à Toulouse, qui s'atténue de 10 à $20 \%$ vers le littoral et la montagne;
- les nappes se sont régulièrement abaissées depuis septembre 1988 et atteignent leur plus bas niveau depuis 20 ans ;

- les débits des rivières sont les plus faibles constatés en cette période hivernale depuis 20 à 50 ans, ils correspondent aux débits d'étiage estival.

L'état des réserves est donné sur la figure de la page suivante.

Compte tenu de la précocité de cette sécheresse 1989 qui débute dès l'hiver, et craignant que cette situation perdure et rende l'été difficile, le 16 février 1989, le Délégué de Bassin Adour-Garonne réunit les Administrations et Organismes publics concernés.

Les prévisions météorologiques ne pouvant se faire audelà de 10 jours, il eût été ambitieux, pour le groupe, d'envisager ce que serait la situation à l'été 1989.

Mais on pouvait affirmer que des pluies même abondantes ne suffiraient pas à rétablir une situation normale.

Les premières actions furent donc d'informer les organisations agricoles des risques de pénurie, afin d'orienter suffisamment tôt les cultures, et d'attirer la vigilance de chacun afin de limiter tout risque de pollution.

Le "groupe de suivi " se réunissant à un rythme bimensuel, examinait les différents scénarios possibles pour l'été.

Ainsi, l'analyse des 13 hivers les plus secs enregistrés à Bordeaux-Mérignac et Toulouse-Blagnac montre que la récupération du déficit pluviométrique par les apports de printemps ne s'est produite que deux fois sur 13 à Bordeaux et trois fois sur 13 à Toulouse et que le déficit global des années concernées reste de $20 \%$ de la normale.

Il y eut des pluies notables fin février et début avril. Ces pluies se sont traduites par une remontée brutale mais éphémère des cours d'eau, les niveaux redescendant audessous du niveau normal après la pluie.

Le niveau des nappes ne remontant pas de façon très nette, les débits des sources restant inférieurs aux normales, l'évapotranspiration reprenant de façon active en avril, tout laissait supposer que la situation en été serait critique. Cependant, ce constat doit être plus ou moins modulé en fonction des Régions, les plus touchées étant MidiPyrénées et Poitou-Charentes.

Les effets de cette sécheresse, en l'absence de pollution accidentelle, ont été minimisés par la saison à laquelle elle s'est déroulée :

- l'irrigation n'a pas démarré ;

- la température de l'eau est basse.

En mars 1989, la situation était la suivante:

- le déficit pluviométrique se maintient ;

- le déficit du stock neigeux est de $50 \%$;

- le niveau des nappes reste bas;

- le débit des sources se maintient à son plus bas niveau connu, alors que l'on constate habituellement une recharge à cette période ; 


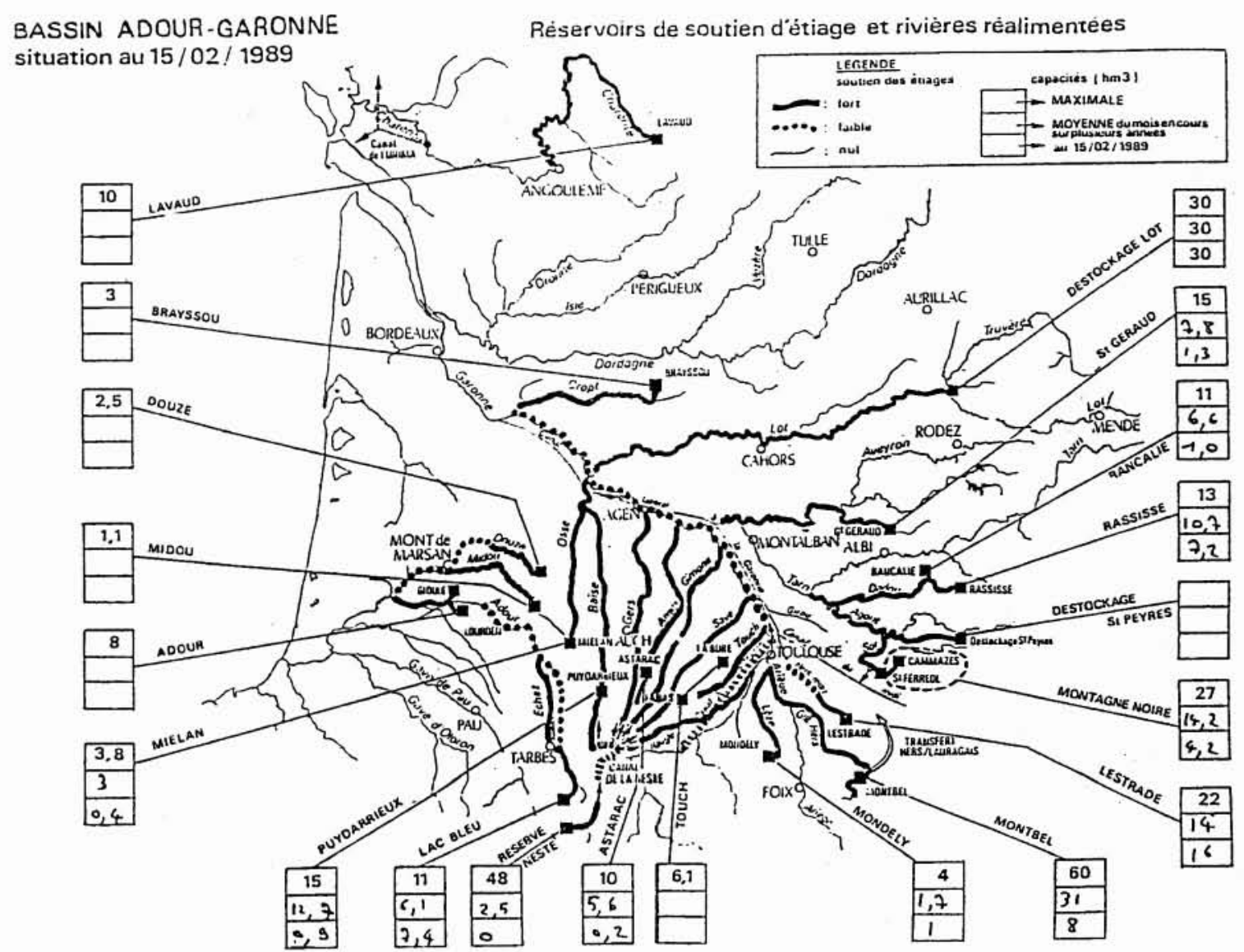

Etat des réserves.

- les débits des cours d'eau restent inférieurs à la moitié du débit de référence ;

- les réserves accusent un déficit croissant sauf pour les réserves EDF du massif Pyrénéen.

Ainsi donc toute une série d'actions fut envisagée afin de garantir au mieux le remplissage des réserves et de prévenir une situation critique estivale.

- Février 1989: Information auprès des Chambres régionales d'agriculture ; demande d'arrêt ou de limitation de la production d'électricité à partir des réserves ; suppression du chômage du canal de la Neste pour 1989, afin d'améliorer le remplissage des barrages de Côteaux du système Neste ; report du chômage au canal de Saint-Martory à l'automne 1989; information des Préfets de Région et de Département ; information aux Services de police des eaux de l'ensemble du bassin ; avancement du chômage du canal du Midi.

- Mars 1989: Suggestion aux Préfets de Région de constituer des groupes régionaux de suivi de la sécheresse autour du SRAE, compte tenu de la diversité de la situation en fonction des régions; EDF diffère la vidange du barrage d'Oule utilisé dans le cadre du système Neste; demande aux Préfets de Département de Midi-Pyrénées de constituer des groupes départementaux de suivi de la sécheresse ; limitation du gabarit sur le canal du Midi alimenté par les réservoirs de la Montagne Noire pendant la période de Pâques.

- Avril 1989: Devant la faiblesse des réserves de la Montagne Noire et vu les prévisions des capacités disponibles pour l'été, il est décidé que le canal du Midi sera fermé entre Naurouze et Carcassonne ; un appel à la vigilance pour la préservation de la qualité de l'eau auprès de l'ensemble des usagers est formulé.

Puis, à la suite de la création du groupe interministériel de suivi de la sécheresse et de la mobilisation des départements, le rôle du groupe de bassin s'est quelque peu modifié. Il s'agissait :

- de rendre compte régulièrement de la situation au Préfet Coordonnateur de bassin et au secrétariat d'Etat à l'Environnement ;

- d'informer et sensibiliser les Préfets et Services de police des eaux de l'ensemble des départements du bassin par 
l'envoi d'un compte rendu bimensuel faisant le point de la situation et de son évolution ;

- synthétiser les informations en provenance des départements, régions ou Ministères et d'en assurer une large diffusion ;

- recenser les mesures prises dans chaque département afin de coordonner les actions de l'Administration.

Ces mesures ont touché de nombreux secteurs d'activité, notamment l'agriculture, le tourisme, la navigation et l'eau potable.

Les premières mesures concernant la limitation de consommation en eau potable ont été prises en juin 1989 sur les secteurs alimentés par la Montagne Noire et se sont généralisées par la suite.

Les mesures touchant l'agriculture furent diverses. Elles ont surtout consisté en l'établissement de tours d'eau en concertation avec la profession agricole. Les irrigations ont été interdites le 16 août en Charente-Maritime et le 15 septembre pour la Garonne en Midi-Pyrénées.

L'arrêt des irrigations a été retardé grâce aux lâchures provenant des réserves EDF et qui font suite aux négo- ciations entre les Ministères concernés et EDF pour l'achat de $40 \mathrm{Mm}^{3}$ pour Midi-Pyrénées.

Un bilan sommaire et provisoire établi fin septembre 1989 fait apparaître que pour l'agriculture les pertes sont évaluées en Midi-Pyrénées à $30 \%$ à $40 \%$ pour les cultures d'été non irriguées et à 10 à $15 \%$ pour les cultures irriguées. Pour les fourrages, les pertes importantes sont proportionnelles au déficit pluviométrique. Mais si l'on a une première estimation des pertes de rendement agricole, il sera plus difficile d'évaluer les pertes des autres usagers tant pour l'alimentation en eau potable et la navigation, que pour la faune et la flore qui ont vécu et vivent encore, au moment de la rédaction de ces lignes, dans un équilibre fragile.

Fin septembre 1989 et depuis 14 mois, le déficit pluviométrique continue de s'accentuer. Cela confirme une étude statistique établie par la Météorologie nationale à Toulouse et qui montre que 8 années sur 10, un été sec est suivi d'un automne sec.

Jusqu'à quand durera ce déficit, nous l'ignorons mais 1989 restera gravée dans les mémoires au même titre que 1949 qui fut une année particulièrement sévère.

\section{Conclusion}

L'examen des préoccupations du "groupe de suivi de la sécheresse 1989 " m'amène à faire les remarques suivantes :

Les premiers soucis du groupe étaient de sensibiliser les différents usagers, surtout agricoles, et d'essayer d'augmenter les réserves de soutien d'étiage disponibles pour l'été.

Sensibilisation des usagers: L'information ciblée vers le secteur agricole au travers des Chambres Régionales et Départementales de l'Agriculture et de la presse agricole n'a pas entraîné la mobilisation que nous escomptions. L'idée selon laquelle :

«s'il ne pleut pas, cela ne va pas durer, on aura un printemps pourri " a freiné toute action et se trouve renforcé par le fait que la marge de manœuvre pour la modification du choix des cultures semble être faible.

Dans le bassin Adour-Garonne, les usagers semblent s'émouvoir du manque d'eau surtout en avril après la fermeture du canal du Midi compte tenu de la faiblesse des réserves de la Montagne Noire.

En Midi-Pyrénées, le canal du Midi s'il est présent dans la vie quotidienne des gens est un ouvrage mythique qui peut soulever des montagnes.

Ainsi, dans la plupart des secteurs la sensibilisation réelle des usagers a débuté deux mois après les premiers travaux du groupe.
Augmentation des réserves: Dans la législation nous n'avons pas trouvé d'article de loi nous permettant de réduire les débits réservés pour augmenter les réserves.

L'article 45 de la loi du 22 juillet 1987 ne permet pas de modification temporaire des débits réservés propre à favoriser la reconstitution partielle des réserves en eau.

De toute façon, la mise en œuvre des dispositions de l'article précité est subordonnée à la déclaration d'état de sécheresse grave qui ne peut avoir lieu qu'en cas de pénurie mettant en péril l'alimentation en eau potable des populations.

Ceci présuppose une série d'actions pour lutter contre les effets de la sécheresse : mesures de police des eaux, mesures de police des rejets, constatation des infractions, mesures de police municipale.

Ainsi, les possibilités d'action de l'Administration en matière de prévention de la pénurie semblent limitées.

De plus, la législation porte essentiellement sur les eaux superficielles. En effet, pour les eaux souterraines les prélèvements supérieurs à $8 \mathrm{~m}^{3} / \mathrm{s}$ ne sont soumis qu'à l'obligation de simple déclaration.

Pourtant, en cas de pénurie, il faut raisonner globalement car les relations entre nappes et rivières font que tout est lié. Les usagers qui sont contraints de limiter leurs pompages en rivière doivent être également contrôlés pour les pompages en nappe. 
L'Etat ne peut que répartir les eaux entre les diverses catégories d'usagers sans qu'il soit possible de fixer les débits par utilisateur individuel, aussi important soit-il. Aussi, l'établissement de règlement d'eau n'a-t-il que peu de soutien juridique.

En cas de limitation des irrigations, il nous semble que la solution la plus efficace pour améliorer le difficile exercice de contrôle des prises d'eau soit un tour d'eau par commune.

Une autre action peut être envisagée lors de l'autorisation de prise d'eau importante. C'est l'établissement par le pétitionnaire, en aval de la prise, d'un système de contrôle avec courbe hauteur-débit associé, qui permettra aux unités mobiles du Service de police des eaux de contrôler rapidement le débit réservé.

Le souci du groupe a également été d'envisager suffisamment tôt les mesures et de préparer leur mise en œuvre. Il m'apparaît important que ces mesures soient élaborées en dehors des périodes de crise. Elles doivent être modulables en fonction de la gravité de la situation et pourront être déclenchées suivant une méthodologie semblable au schéma établi pour la protection des populations. Les seuils des différentes mesures à prendre seront déterminés au regard des expériences passées et chaque seuil sera accompagné de mesures portant sur tout au partie de l'usage de l'eau élaborées conjointement avec les différents usagers.

Si les périodes de crise sont parfois difficiles à vivre, elles sont néanmoins riches en enseignement. A cet égard, le groupe de Bassin, dans la mise en place d'une gestion de crise, s'attache à deux critères essentiels :

Relation quantité-qualité: Solidarité en quantité mais aussi en qualité. Le but est non seulement de gérer au mieux l'eau en quantité mais également de gérer de l'eau de bonne qualité.

N'oublions pas que par usager, si l'on pense aux usagers de surface, il faut prendre en considération les usagers aquatiques constitués par la faune et la flore.

L'effort doit donc porter sur la quantité et la qualité, et ce d'autant plus, qu'une pollution accidentelle en période d'étiage peut se retrouver par transfert dans les nappes alluviales et hypothéquer sérieusement le devenir de cellesci pour plusieurs années.

\section{Composition du groupe de suivi de la sécheresse 1989}

Ce « groupe » comprend :

- le Délégué de Bassin Adour-Garonne;

- le Service régional de l'aménagement des eaux de Midi-Pyrénées; - le Service régional de l'aménagement des eaux d'Aquitaine ;

- la Météorologie nationale ;

- la Direction régionale de l'industrie et de la recherche de Midi-Pyrénées ;

- le Service de la navigation de Toulouse ;

- l'Agence financière de bassin Adour-Garonne ;

- EDF ;

- Compagnie d'aménagement des Côteaux de Gascogne ;

- Institution interdépartementale pour l'aménagement hydraulique de la Montagne Noire ;

A ce groupe se joint par la suite :

- le Conseil supérieur de la pêche ;

- le Bureau régional d'études géologiques et minières ;

- la Direction départementale de l'agriculture et de la forêt de la Haute-Garonne.

Pour illustrer cette solidarité, je citerai pour mémoire l'expérience menée actuellement en Charente, sous l'égide de l'Institution interdépartementale pour l'aménagement du fleuve " Charente " : un protocole s'élabore entre l'Etat, l'Institution, l'Agence de l'eau et l'ensemble des usagers afin d'améliorer la ressource en eau et de gérer au mieux les nappes et rivières pour préserver les intérêts de tous, y compris des habitants aquatiques. Il s'agit là d'un engagement solidaire.

Enfin, pour terminer, je dirai que l'idée selon laquelle l'eau est un bien gratuit dont tout le monde peut disposer comme il l'entend est révolue : il nous faut considérer l'eau comme une richesse épuisable, indispensable à la survie de toutes les espèces, humaine, animales et végétales. Notre devoir est de la préserver tant en quantité qu'en qualité pour la transmettre en bon état aux générations futures. 


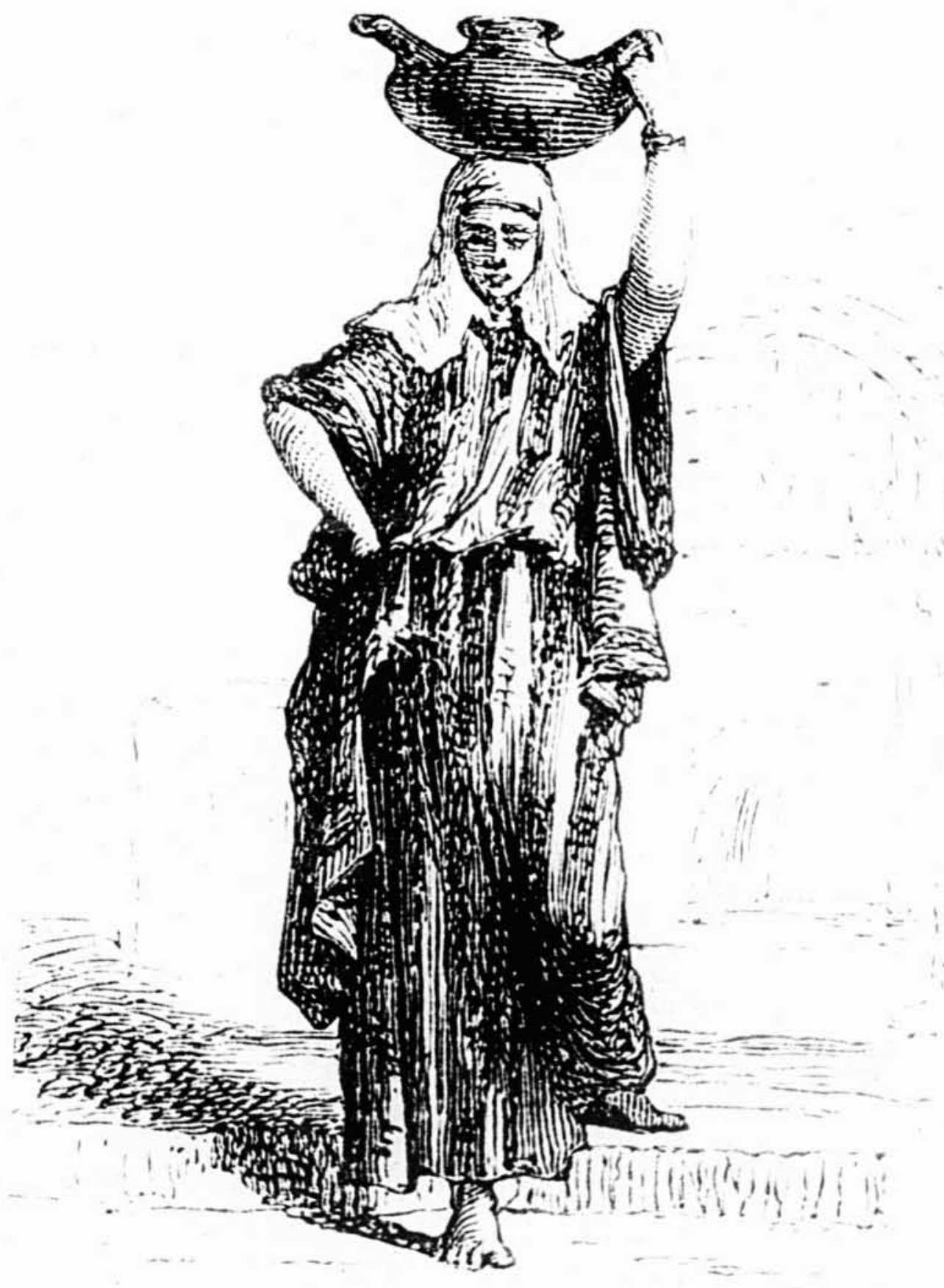

